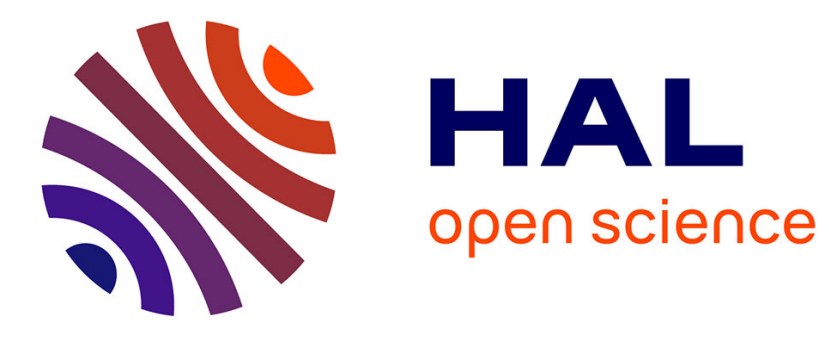

\title{
Stability of Hydrated Clay Layers from Molecular Simulations
}

Túlio Honório, Laurent Brochard, Matthieu Vandamme, Ioannis Stefanou, Siavash Ghabezloo, Michel Bornert

\section{- To cite this version:}

Túlio Honório, Laurent Brochard, Matthieu Vandamme, Ioannis Stefanou, Siavash Ghabezloo, et al.. Stability of Hydrated Clay Layers from Molecular Simulations. Sixth Biot Conference on Poromechanics, Jul 2017, Paris, France. pp.688, 10.1061/9780784480779.085 . hal-01686230

\section{HAL Id: hal-01686230 \\ https://hal.science/hal-01686230}

Submitted on 20 Jun 2018

HAL is a multi-disciplinary open access archive for the deposit and dissemination of scientific research documents, whether they are published or not. The documents may come from teaching and research institutions in France or abroad, or from public or private research centers.
L'archive ouverte pluridisciplinaire HAL, est destinée au dépôt et à la diffusion de documents scientifiques de niveau recherche, publiés ou non, émanant des établissements d'enseignement et de recherche français ou étrangers, des laboratoires publics ou privés. 


\title{
Stability of hydrated clay layers from molecular simulations
}

\author{
Tulio Honorio, $\mathrm{PhD},{ }^{1}$ Laurent Brochard, $\mathrm{PhD},{ }^{2}$ Matthieu Vandamme, $\mathrm{PhD},{ }^{3}$ \\ Ioannis Stefanou, PhD, ${ }^{4}$ Siavash Ghabezloo, $\mathrm{PhD},{ }^{5}$ and Michel Bornert, $\mathrm{PhD}^{6}$ \\ ${ }^{1}$ Laboratoire Navier, UMR 8205, Ecole des Ponts, IFSTTAR, CNRS, UPE, Champs- \\ sur-Marne, France; e-mail: tulio.honorio-de-faria@enpc.fr \\ ${ }^{2}$ Laboratoire Navier, UMR 8205, Ecole des Ponts, IFSTTAR, CNRS, UPE, Champs- \\ sur-Marne, France; e-mail: laurent.brochard@enpc.fr \\ ${ }^{3}$ Laboratoire Navier, UMR 8205, Ecole des Ponts, IFSTTAR, CNRS, UPE, Champs- \\ sur-Marne, France; e-mail: matthieu.vandamme@enpc.fr \\ ${ }^{4}$ Laboratoire Navier, UMR 8205, Ecole des Ponts, IFSTTAR, CNRS, UPE, Champs- \\ sur-Marne, France; e-mail: ioannis.stefanou@enpc.fr \\ ${ }^{5}$ Laboratoire Navier, UMR 8205, Ecole des Ponts, IFSTTAR, CNRS, UPE, Champs- \\ sur-Marne, France; e-mail: siavash.ghabezloo@enpc.fr \\ ${ }^{6}$ Laboratoire Navier, UMR 8205, Ecole des Ponts, IFSTTAR, CNRS, UPE, Champs- \\ sur-Marne, France; e-mail: michel.bornert@enpc.fr
}

\begin{abstract}
Clays adsorb water following discrete steps in which water is roughly structured in layers. The resulting discrete hydration states can be viewed as phases separated by unstable domains and the change of hydration state can be viewed as a phase transformation. Here, we identify stable basal spacings and the domains of (meta-) stability through the analysis of confining pressure isotherms obtained from molecular simulations performed in grand canonical ensemble. This ensemble mimics drained conditions. Through thermodynamic analysis, we can explain the coexistence of different hydration states at clay particle scale and provide kinetics arguments to the hysteresis observed with respect to traction and compression. The gained insights into the mechanical behavior of the elementary constituents of clays may lead to a better understanding of the behavior of clay-rich rocks and soils.
\end{abstract}

\section{INTRODUCTION}

Clays are layered nanoporous materials containing adsorbed water. This peculiar interaction with water is known to induce large volume changes, which are relevant to a variety of environmental processes and industrial applications. Clays adsorb water following discrete steps in which water is roughly structured in layers, characterizing hydration states, depending on the environmental conditions. The environmental conditions of interest for geological sites or experimental setups are in general described in terms of confining pressure, water pressure and temperature.

In clays, transition between hydration states were treated as phase transformations by different authors (Tambach et al. 2006; Wada et al. 1990). 
Hydration states can be seen as phases separated by unstable domains. Stable states can be viewed as the ones minimizing a given thermodynamic potential of interest. In drained conditions, water in clay interlayer pores is in equilibrium with an external water reservoir. For a porous medium under strain or displacement control, these conditions correspond to a mixed thermodynamic potential composed of the Helmholtz free energy of the solid skeleton plus Grand potential of the water. For clays with different stable hydration states, such potential displays a multi-well configuration. Similar multi-well potentials are encountered in Shape Memory Alloys, in which very peculiar crystallographic properties are known to allow for phase transitions with various stable states coexisting inside the same crystal (Bhattacharya 2003). For clays, similar phase coexistence seems possible at the scale of stacks of layers, generally referred to as clay particle. Clay particle is often identified on the purpose of understanding some aspects of clays macroscopic behavior (Salles et al. 2010). Experimental evidence (e.g.(Ferrage et al. 2010) for XRD results) shows that different hydration states may coexist in clay particles.

Here, we identify stable basal spacings and the domains of (meta-)stability through the analysis of confining pressure isotherms obtained from molecular simulations. Our results explain the coexistence of different hydration states at clay particle scale and provide kinetics arguments, based on the energy barriers to be overcome in transformations, to the hysteresis observed with respect to traction and compression. Further, the stability analysis allows one to draw a phase diagram of the stable hydration state of clays particles as a function of the confining pressure, water pressure and temperature. We present the case of Na-saturated Montmorillonite clay (Na-Mmt) for which Grand-Canonical Monte Carlo simulations are performed to obtain confining pressure isotherms.

\section{THERMODYNAMIC POTENTIALS AND STABILITY}

Thermodynamic potentials at microscopic level. The basic structural units of clays are solid layers intercalated by water and cations. In drained experimental conditions, water in clay interlayer pores is in equilibrium with an external water reservoir. Molecular simulations that mimic these conditions are performed in the so-called Grand Canonical ensemble - here $\left(\mu_{w} V T\right)$, in which the chemical potential of water $\mu_{w}$, the volume $V$, and the temperature $T$ of a system are imposed, while the amount of water, the confining stress and entropy are fluctuating. This ensemble minimizes a mixed thermodynamic potential $\Lambda$ composed of the Helmholtz free energy of the solid skeleton and counterions plus the Grand potential of the water:

$$
\Lambda\left(\mu_{w}, N_{\text {ions }}, N_{\text {sol }}, V, T\right)=F-\mu_{w} N_{w} \text { (1) }
$$

where $N_{w}, N_{\text {ions }}$ and $N_{\text {sol }}$ are the number of water molecules, counterions and species in the solid layers, respectively; $\mathrm{F}$ is the Helmholtz free energy. At the layer scale, the volume $V$ per unit of surface $A$ is equal to the basal spacing $e$, i.e. $V / A=$ $e$. The basal spacing is the sum of the thickness of solid clay layer and the pore width. Let $\lambda$ be the potential $\Lambda$ per unit area: $\lambda \equiv \Lambda / A$. The confining pressure $P$ acting in the direction orthogonal to the solid layers is defined as $P=-\frac{\partial \lambda}{\partial e}$. Thus, the 
evolution of $\lambda$ with respect to changes in basal spacing can be obtained by integrating the confining pressure:

$$
\lambda\left(e, T, \mu_{w}\right)=\lambda\left(e_{0}, T, \mu_{w}\right)-\int_{e_{0}}^{e} P d e
$$

A system in which the confining pressure $P$ is controlled instead of the volume, minimizes a complementary energy, obtained by a Legendre transform:

$$
\Lambda^{*}\left(\mu_{w}, N_{\text {ions }}, N_{\text {sol }}, P, T\right)=\Lambda+P V
$$

Accordingly, the complementary energy per unite area $\lambda^{*}=\Lambda^{*} / A$ is:

$$
\lambda^{*}=\lambda+P e
$$

In layered materials, typical confining pressure isotherms are oscillating curves with decaying amplitudes, Figure 1 (left, right) shows the first oscillations. Figure 1 (left, bottom) and (right, top) illustrate the resulting thermodynamic potential and its complementary potential, respectively.

Phase transformation and stability. Materials with a non-convex potential $\lambda$ are prone to develop a heterogeneous microstructure in order to minimize their total energy. In this section, we show how the coexistence of hydration states and the stability of clays can be understood as the minimization of the mesoscopic energy associated to a clay particle. Mesoscopic energy is the minimization of the total microscopic energy over a given representative volume element (RVE) $\Omega$, with imposed average strain $\varepsilon$ (or equivalently average basal spacing $\langle e\rangle$ ) on the boundary $\partial \Omega$ of the REV:

$$
\hat{\lambda}\left(\langle e\rangle, T, \mu_{w}\right) \equiv \underbrace{\inf _{\Omega}}_{\langle e\rangle \text { on } \partial \Omega} \int_{\Omega} \lambda\left(e, T, \mu_{w}\right) d \mathrm{x}
$$

If the solution of this minimization problem corresponds to a homogeneous basal spacing $\langle e\rangle=e$; this basal spacing is stable. $\hat{\lambda}$ is the convex hull of $\lambda$ and includes all the possible stable states of $\Omega$ under displacement control. In other portions of $\lambda$, a deformation (or basal spacing) $e_{C}$ laying in-between two stable states $e_{A}$ and $e_{B}$, is accommodated in the particle by mixing the stable states:

$$
e_{C}=f_{A} e_{A}+\left(1-f_{A}\right) e_{B}
$$

with $\left.\mathrm{f}_{\mathrm{A}} \in\right] 0,1$ [ being the volume fraction of material in state $\mathrm{e}_{A}$. Figure 2 shows a schematic representation of the mixture of stable states. Figure 1 (bottom left) represents the microscopic energy $\lambda$ and its convex envelop (red dashed) is the mesoscopic energy $\hat{\lambda}$. The stable phase transitions states are identified, not by the local minima of $\lambda$, but by the red points. Stable phase transition occurs at constant confining pressure (see dashed line in Fig. 1 (left, top)). In terms of complementary energy $\lambda^{*}$, the phase transition basal spacings correspond to the local minima and are a function of the pressure $P$ (see Fig. 1(top, right)). Stable phase transition occurs for the pressure at which the two minima are equal; the other situations are meta-stable.

The minimization of the mesoscopic energy by fine-mixture of stable states leads, a priori, to infinitely fine microstructures (Bhattacharya 2003). Specific considerations can be made in order to define a minimal characteristic length of each "phase". In clays systems, the minimal characteristic length is the $e$ itself. Contrary to SMA (and under the assumptions of 1D kinematics for perfectly parallel and rigid particles), no constraints of coherence and compatibility are necessarily imposed 
onto the interfaces between phases in clay layers. At the mesoscale, the energies defined here do not account for specific phenomena related to the interfaces between neighbor clay particles. Such phenomena can further penalize the energy barrier to be overcome in phase transition.
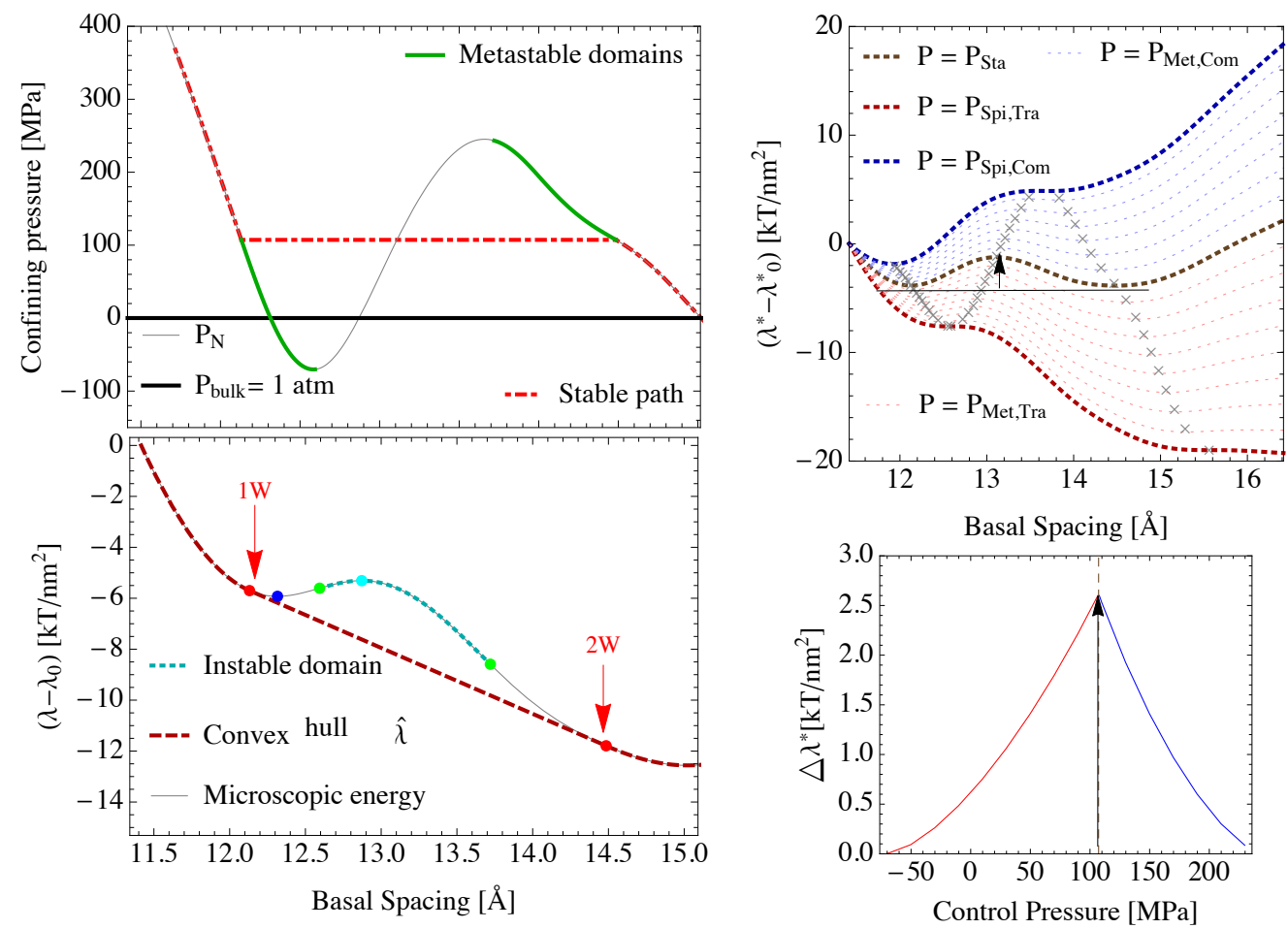

Figure 1. Confining pressure isotherm (left, top), thermodynamic potentials $\lambda$ (left, bottom) and $\lambda^{*}$ (right, top) and energy barriers (right, bottom) $\Delta \lambda *$ for Na-

Mmt at $300 \mathrm{~K}$ and $1 \mathrm{~atm}$ based on (Carrier 2013). In (left, bottom) the red dashed line represent the convex hull of the energy $\lambda$. In (right, bottom) the energy barriers for $1 \mathrm{~W} / 2 \mathrm{~W}$ transitions are displayed for the stable (black arrow) and metastable states (red curve for $1 \mathrm{~W} \rightarrow 2 \mathrm{~W}$; blue curve for $2 \mathrm{~W} \rightarrow 1 \mathrm{~W}$ )

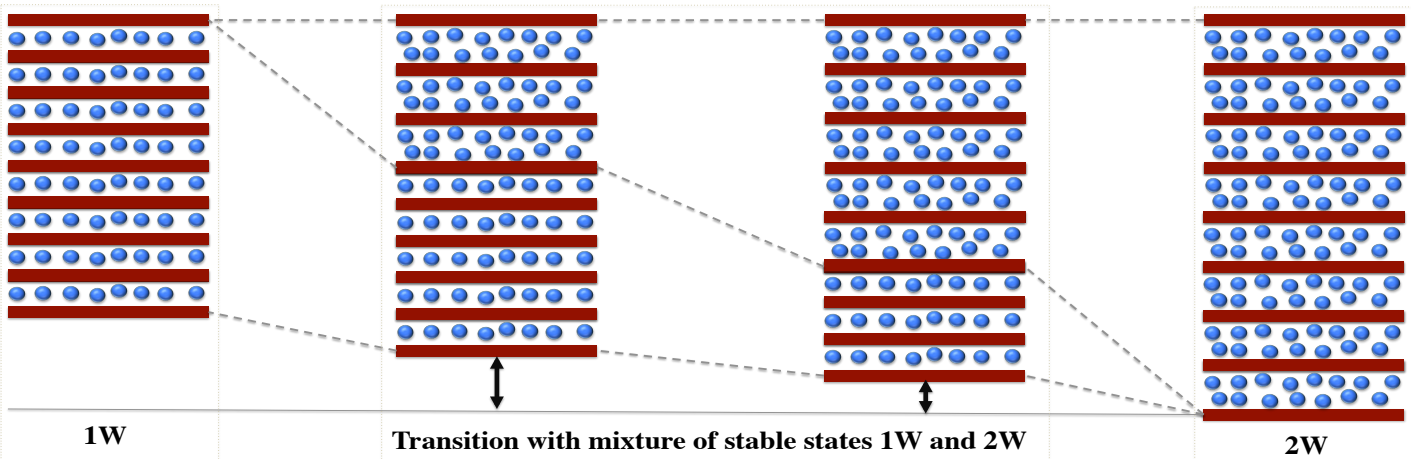

Figure 2. Sketches of configurations corresponding to stable and arbitrary $e$.

Metastability and energy barriers. The consideration of metastability is necessary to capture the hysteresis in clays behavior. The limits (spinodal decomposition) of 
metastable zones are defined by the basal spacings in which $\partial^{2} \lambda / \partial e^{2}=0$. The energy barrier to be overcome in phase transformation between two stable states is obtained by means of complementary energy $\lambda^{*}$. Tambach et al. (2006) reported energy barriers for Na-Mmt in the order of few $k T / \mathrm{nm}^{2}$. Clay layers present lateral dimensions ranging from 100 to $1000 \mathrm{~nm}$ (Suter et al. 2007). The corresponding total energy barrier associated to transition in a clay layer is in favor of metastability. Considering the size of clay layers, transition presumably involves bending of the layers in such a way that only small portions of the layers overcome the energy barrier. It is noteworthy, though, that even if the bending occurs over short length (e.g., a few $\mathrm{nm}$ ), the energy barrier seems sufficient for metastability to hold. As can be seen in Figure 1(right), for $P$ equal to the pressure of stable phase transformation $P_{S t a}$, both stable states have the same $\lambda^{*}$. For a $P$ equal to the pressure at the spinodal points $P_{S p i, T r a}$ or $P_{S p i, C o m}$ (for traction and compression, respectively), the energy barrier is zero $\left(\operatorname{sign}(\partial \lambda / \partial e)\right.$ is constant, i.e. $\lambda^{*}$ is monotonic), as can be seen for the topmost and bottommost curves in Figure 1(right). The energy barriers $\Delta \lambda^{*}$ of the (intermediary) metastable states provides information on the prevalence of a given hydration state in a given loading as well as kinetic information. For $\Delta \Lambda^{*}$ with the order of magnitude of the thermal fluctuations $(\approx k T)$, small perturbations in the system can lead to a transition to a state with lower energy. Following transition state theory (Truhlar et al. 1985), the time necessary to overcome a given free energy barrier $\left(\Delta \Lambda^{*}\right.$, here) scales with $\exp \left[-\Delta \Lambda^{*} /(k T)\right]$. The prevalence of (meta-)stable states can be, thus, compared according to the loading type.

\section{MOLECULAR SIMULATIONS OF NA-MMT AND STABILITY ANALYSIS}

Models and methods. For molecular simulations, the Mmt super cell was obtained by replicating the layers obtained from pyrophyllite structure (as probed by XRD). Details on the substitutions of metal atoms in the solid structure are provided in (Carrier 2013). The electrical charge of the layers is compensated by the appropriated number of $\mathrm{Na}$ cations in the interlayer space. The resulting structural formula is $\mathrm{Na}_{6}\left[\mathrm{Si}_{62} \mathrm{Al}_{2}\right]\left[\mathrm{Mg}_{4} \mathrm{Al}_{28}\right] \mathrm{O}_{160}(\mathrm{OH})_{32} \cdot \mathrm{nH}_{2} \mathrm{O}$. The interactions between atoms are described by Lennard-Jones and Coulomb potentials. Lennard-Jones parameters and partial charges are taken from ClayFF force field (Cygan et al. 2004) combined with SPC/E water model (Berendsen et al. 1987). Lennard-Jones cross relations are obtained using Lorentz-Berthelot mixing rules. For short-range dispersive interactions, a cutoff distance of $8.9 \AA$ is adopted. For long-range electrostatic interactions, Ewald's summation is employed with precision of $10^{-4}$. Simulations were run with periodic boundary conditions. Towhee (Martin 2013) Monte Carlo package was used to perform CBMC (Configuration-Bias Monte Carlo) simulations. In this paper, we consider three temperatures, 300, 400 and $500 \mathrm{~K}$, at a target confining pressure of $20 \mathrm{MPa}$. To determine the chemical potential $\mu_{w}$ corresponding to these temperatures and confining pressure, we run simulations of bulk water in grand canonical (GC) and canonical ensembles (in MD with LAMMPS (Plimpton 1995), in this case). Water molecules are kept rigid and we neglect their rotational degree of freedom. In NVT simulation, $\mu_{w}$ is obtained from thermodynamic integration of Gibbs-Duhem equation from the liquid branch of 
coexistence curve of SPC/E (NIST 2014). The outputs of both simulations are in fair agreement regarding the identification of the range of $\mu_{w}$ associated to each one of the three temperatures for confining pressures varying from 1 to $100 \mathrm{MPa}$. However, in GC simulations of bulk water, large variations of the confining pressure with respect to an imposed $\mu_{w}$ are observed (see e.g. Fig. 1 in (Smith et al. 2006)). More precise simulations are ongoing in order to validate the relation between $\mu_{w}$, confining pressure and temperature. CBMC simulations are run to fill clay interlayer space with water according to different basal spacings in response to the determined $\mu_{w}$. Clay layers are also kept rigid. Only the number of water molecules was allowed to evolve in the simulation box, whilst counterions were treated in the canonical ensemble (as in Eq. (1)).

Results at 300, 400 and $500 \mathrm{~K}$. Figure 3 shows the confining pressure obtained from GCMC simulations of Na-Mmt as a function of the basal spacing. The smoothed data (through Savitzky-Golay algorithm) used in stability analyses is also displayed. Figure 4 shows the convex hull of the energy for the three temperatures. The amplitudes of oscillations decrease with the temperature. The pressures and energies barriers $\Delta \lambda^{*}$ in phase transitions, following the stable path, are presented in Table 1. For $0 \mathrm{~W} / 1 \mathrm{~W}$ transitions, the energy barriers decrease with the temperature. Further investigation is necessary to validate the nonlinear evolution of the stable $\Delta \lambda^{*}$ with the temperature. Also, the effect of box size needs to be analyzed to make sure that the differences between target confining pressure and the pressure obtained in clays GCMC simulations at large basal spacing are not an artifact. Figure 5 shows the energy barriers also in metastable conditions for $0 \mathrm{~W} / 1 \mathrm{~W}$ transitions.
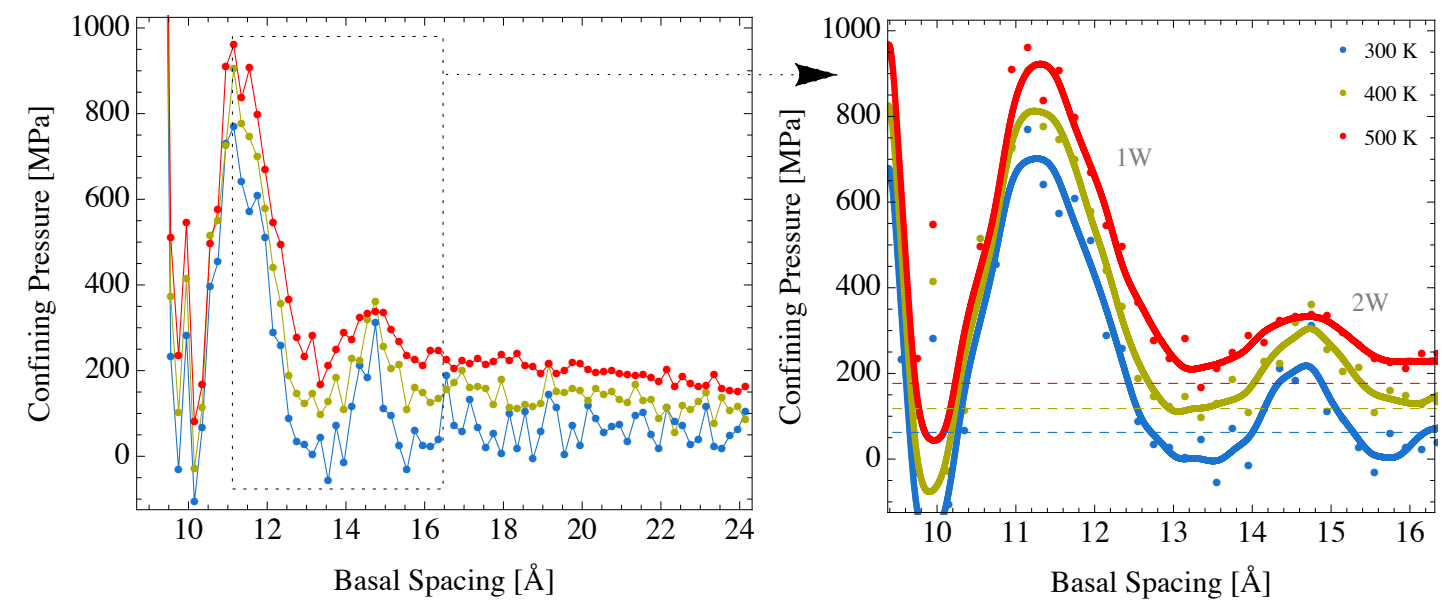

Figure 3. Confining pressure isotherms from CBMC simulations of Na-Mmt at 300,400 and $500 \mathrm{~K}$ (left) and smoothed data (right).

The energy barrier associated to a control pressure corresponding to the pressure of stable phase transition is higher than the energy barriers associated to metastable states. The (metastable) energy barrier decreases more rapidly with a given increment of pressure favoring the $1 \mathrm{~W} \rightarrow 0 \mathrm{~W}$ transition with respect to the $0 \mathrm{~W} \rightarrow 1 \mathrm{~W}$ transition. Thus, for a given surface area of clay layer, the change 
$1 \mathrm{~W} \rightarrow 0 \mathrm{~W}$ is expected to occur more rapidly than $0 \mathrm{~W} \rightarrow 1 \mathrm{~W}$. For instance at $300 \mathrm{~K}$, the ratio $\exp \left[-\Delta \Lambda^{*}{ }_{1 W \rightarrow 0 W} /(k T)\right] / \exp \left[-\Delta \Lambda^{*}{ }_{0 W \rightarrow 1 W} /(k T)\right]=2.8$ for an increment of pressure of $100 \mathrm{MPa}$ with respect to the stable transition pressure towards the metastable domain.
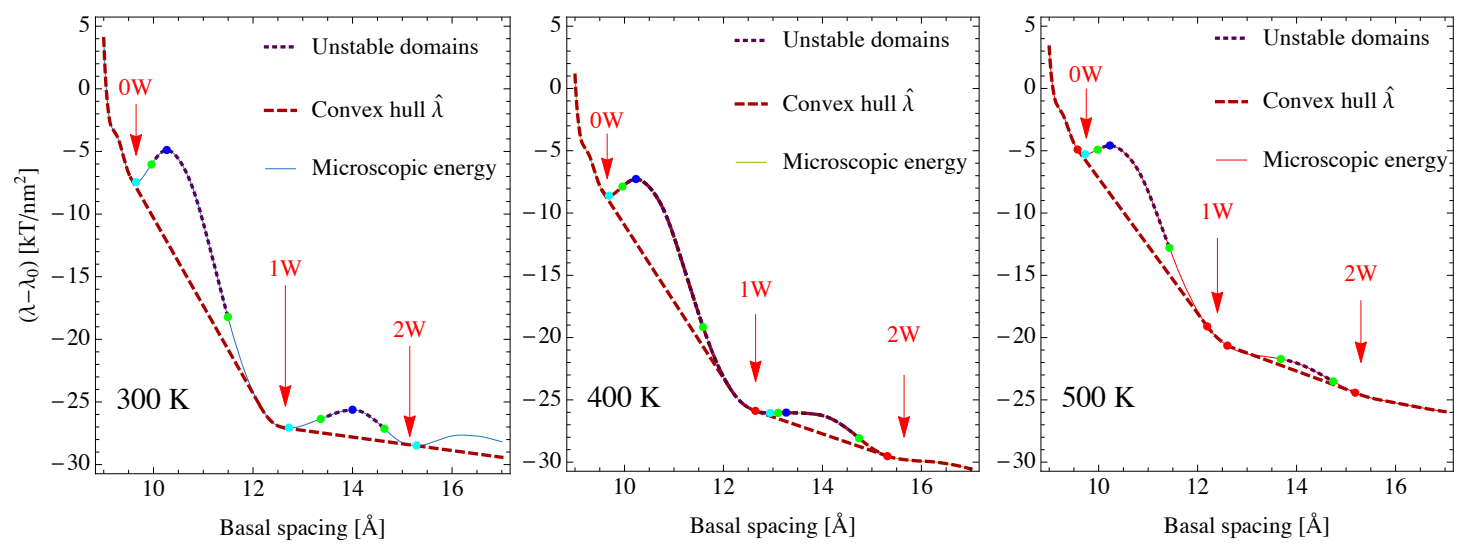

Figure 4. Convex hull of the $\lambda$ for Na-Mmt at 300, 400 and $500 \mathrm{~K}$.

Table 1: Pressure and energy barrier at

\begin{tabular}{|c|c|c|c|c|}
\multicolumn{5}{|c|}{ stable transitions } \\
\hline \multicolumn{2}{|c|}{ T $[\mathbf{K}]$} & $\mathbf{3 0 0 K}$ & $\mathbf{4 0 0} \mathbf{K}$ & $\mathbf{5 0 0} \mathbf{~ K}$ \\
\hline Pressure & $O W / 1 W$ & 290.0 & 339.1 & 374.0 \\
\cline { 2 - 5 }$[\mathbf{M P a}]$ & $1 W / 2 W$ & 22.3 & 75.0 & 100.3 \\
\hline$\Delta \boldsymbol{\lambda}^{*}$ & $O W / 1 W$ & 8.29 & 6.26 & 5.03 \\
\cline { 2 - 5 }$\left[\mathbf{k T} / \mathbf{n m}^{2}\right]$ & $1 W / 2 W$ & 2.18 & 1.48 & 0.72 \\
\hline
\end{tabular}

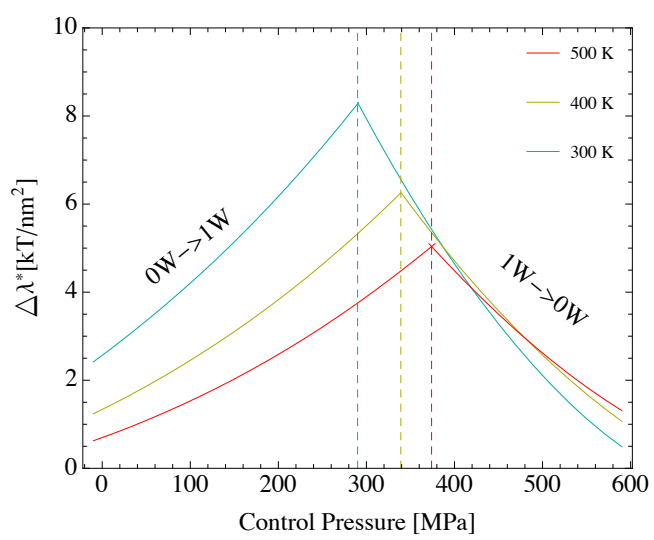

Figure 5: Energy barriers at stable and metastable $0 \mathrm{~W} / 1 \mathrm{~W}$ transitions.

\section{CONCLUSION AND PERSPECTIVES}

The stability analysis presented in this paper explains the coexistence of different hydration states at clay particle scale and provides kinetics arguments to the hysteresis observed with respect to traction and compression. Further developments include the construction of a phase diagram of the stable hydration state of clays particles as a function of the confining pressure, water pressure and temperature. Such information provides interesting insights into the mechanical behavior of the elementary constituents of clays, which may be of interest for a better understanding of the thermomechanical behavior of clay-rich rocks and soils.

Acknowledgments. The financial support of the French National Research Agency (ANR) through the project TEAM2ClayDesicc (ANR-14-CE05-0023-01) is gratefully acknowledged. 


\section{REFERENCES}

Berendsen, H. J. C., Grigera, J. R., and Straatsma, T. P. (1987). "The missing term in effective pair potentials." The Journal of Physical Chemistry, 91(24), 62696271.

Bhattacharya, K. (2003). Theory of martensitic microstructure and the shapememory effect.

Carrier, B. (2013). "Influence of water on the short-term and long-term mechanical properties of swelling clays : experiments on self-supporting films and molecular simulations." phdthesis, Université Paris-Est.

Cygan, R. T., Liang, J.-J., and Kalinichev, A. G. (2004). "Molecular Models of Hydroxide, Oxyhydroxide, and Clay Phases and the Development of a General Force Field." The Journal of Physical Chemistry B, 108(4), 12551266.

Ferrage, E., Lanson, B., Michot, L. J., and Robert, J.-L. (2010). "Hydration Properties and Interlayer Organization of Water and Ions in Synthetic NaSmectite with Tetrahedral Layer Charge. Part 1. Results from X-ray Diffraction Profile Modeling." The Journal of Physical Chemistry C, 114(10), 4515-4526.

Martin, M. G. (2013). "MCCCS Towhee: a tool for Monte Carlo molecular simulation." Molecular Simulation, 39(14-15), 1212-1222.

NIST. (2014). "SAT-TMMC: Liquid-Vapor coexistence properties - SPC/E Water." $<$ http://www.nist.gov/mml/csd/informatics_research/spce_water_sat_lrc.cfm\# refs $>$.

Plimpton, S. (1995). "Fast Parallel Algorithms for Short-Range Molecular Dynamics." Journal of Computational Physics, 117(1), 1-19.

Salles, F., Bildstein, O., Douillard, J. M., Jullien, M., Raynal, J., and Van Damme, H. (2010). "On the Cation Dependence of Interlamellar and Interparticular Water and Swelling in Smectite Clays." Langmuir, 26(7), 5028-5037.

Smith, D. E., Wang, Y., Chaturvedi, A., and Whitley, H. D. (2006). "Molecular Simulations of the Pressure, Temperature, and Chemical Potential Dependencies of Clay Swelling." The Journal of Physical Chemistry B, 110(40), 20046-20054.

Suter, J. L., Coveney, P. V., Greenwell, H. C., and Thyveetil, M.-A. (2007). "LargeScale Molecular Dynamics Study of Montmorillonite Clay: Emergence of Undulatory Fluctuations and Determination of Material Properties." The Journal of Physical Chemistry C, 111(23), 8248-8259.

Tambach, T. J., Bolhuis, P. G., Hensen, E. J. M., and Smit, B. (2006). "Hysteresis in Clay Swelling Induced by Hydrogen Bonding: Accurate Prediction of Swelling States." Langmuir, 22(3), 1223-1234.

Truhlar, D. G., Isaacson, A. D., and Garrett, B. C. (1985). "Generalized transition state theory." Theory of chemical reaction dynamics, 4, 65-137.

Wada, N., Hines, D. R., and Ahrenkiel, S. P. (1990). "X-ray-diffraction studies of hydration transitions in Na vermiculite." Physical Review B, 41(18), 1289512901. 